\title{
Distributed Measurement for the Process Industry
}

\author{
Bierweiler, Thomas ${ }^{1}$, Ens, Wolfgang ${ }^{2}$, Kölsch, Joachim ${ }^{2}$, Sass, Frank ${ }^{3}$, Dr. von Dosky, Stefan ${ }^{1}$ \\ 1 Siemens AG, Process Industries and Drives Division, Technology and Innovations, PD TI AT 2, \\ Oestliche Rheinbrueckenstr. 50, 76187 Karlsruhe, Germany, \\ ${ }^{2}$ Siemens AG, Process Industries and Drives Division, Process Automation, Process Instrumentation, \\ Oestliche Rheinbrueckenstr. 50, 76187 Karlsruhe, Germany \\ ${ }^{3}$ Siemens AG, Process Industries and Drives Division, Process Automation, Sales, Oestliche \\ Rheinbrueckenstr. 50, 76187 Karlsruhe, Germany \\ thomas.bierweiler@siemens.com
}

\begin{abstract}
:
Capturing more information from equipment installed in a process plant is an important trend in the field of process instrumentation. Measuring the process temperature with high spatial resolution to observe and track the distributed reaction intensity is becoming increasingly important. Temperature gradients and dangerous hot spots should be localized using multipoint temperature sensors. An optical sensor system with fiber Bragg grating (FBG) technology is the preferred approach. A factory calibrated plug \& play system has been developed. The wide temperature range from $-230{ }^{\circ} \mathrm{C}$ to $500^{\circ} \mathrm{C}$, the good accuracy, excellent long term stability - better than class B (IEC 60751) -, the enormous amount of sensors (up to 192) and long, highly bendable lances mean that this nextgeneration temperature measuring system offers many advantages.
\end{abstract}

Key words: distributed temperature measurement, multipoint temperature sensors, fiber Bragg grating technology, femtosecond-laser-written fiber Bragg gratings

\section{More Information about the Reactor: Distributed Temperature Measurement}

Capturing more information from equipment installed in process plants is an important trend in the field of process instrumentation for improving operation. To keep installation costs at a reasonable level, new measurement systems are required that perfectly fit in the process vessel.

The future of the chemical industry with its highly developed and cost-intensive facilities is closely linked to innovative processes and plant technology. The requirements relating to admissions and energy costs lead to a modernization of these "old" facilities. Multiple educts to feed into a reactor, special temperature gradients and high pressures are often attributes of new processes. Furthermore, there is less specific knowledge of plant operation available as profitability means that the development times are becoming shorter and shorter. This development involves the product itself, the process and the plant design from the initial laboratory status to a full-size production plant. This is why it makes sense to measure the process temperature for instance with high spatial resolution to observe and track the distributed reaction intensity. Temperature gradients and dangerous hot spots are to be localized using multipoint temperature sensors. Presently, stainless steel tubes accommodating a few thermocouples or resistance thermometers are generally being used. Typically, such a multipoint sensor is several meters long, has a diameter of about 6 to $12 \mathrm{~mm}$ and is equipped with $8-12$ thermocouples or resistance temperature devices (RTDs). To observe and control a larger reactor, a customer must use a multitude of sensors. Cabling conventional multipoint sensors such as these is complex, and these types of lances are subject to mechanical deformation, especially during installation and removal. Furthermore, multipoint temperature measurement technology is not very versatile and cannot address new applications [1].

\section{Fiber Bragg Grating Technology for} Distributed Temperature Measurement

In the field of fiber optic sensors a lot of progress has been made in recent years, and experience has been gathered based on many field tests. FBG technology is the preferred approach because of its high signal-to-noise ratio. Although sensitive systems were available in the past, they were not comparable with rugged conventional electrical temperature sensors, which are stable over the long-term. Today advances, mainly relating to the electro- 
optical units (interrogators) in terms of performance, minimize the environmental impact on the measurement results to a level where it can be ignored. The sensors (lances) with lengths of up to 40 meters are comparatively small, rugged and equipped with a small plug for just one optical fiber. Thorough investigations were made to improve the calibration and the long-term stability of the system at elevated operating temperatures. The system configuration in the firmware of the interrogator was improved to hide the optical complexity. Today, we have factory-calibrated plug \& play systems which can easily be installed and operated by a customer who is familiar with conventional temperature sensors. The wide temperature range from $-230{ }^{\circ} \mathrm{C}$ to $+500{ }^{\circ} \mathrm{C}$ (which has been verified), the good accuracy with factory calibration, excellent longterm stability (better than class B) for resistance thermometers (IEC 60751), the enormous number of sensors (up to 192), a PROFIBUS DP and an Ethernet interface as well as long and highly bendable lances mean that this next generation temperature measurement system offers many advantages. The small geometry of the lance with its rugged plug allows the sensor to be an integral part of the process vessel (Fig. 1).

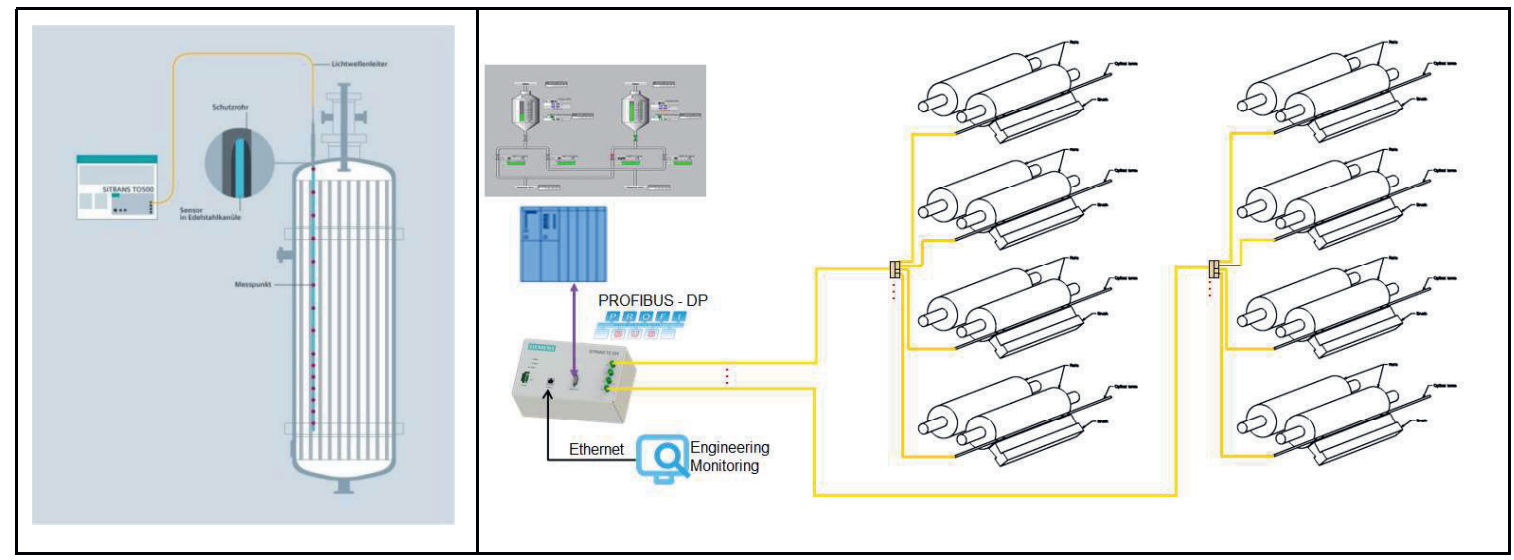

Fig. 1. System set-up for a mid-sized installation (e.g. for a reactor) (left) and for an extremely large installation (e.g. a grain mill) (right)

\section{Temperature Measurement down to $30 \mathrm{~K}$}

Investigations using a cryostat at the Institute for Technical Physics (ITEP), KIT-Nord, Germany, at temperatures down to $8 \mathrm{~K}$ using different types of FBGs (both phase mask gratings of type I and gratings inscribed with femtosecond lasers of type II) [2] show sufficient sensitivity for temperature measurements starting at $30 \mathrm{~K}$. In the temperature range from $8 \mathrm{~K}$ to $15 \mathrm{~K}$, no changes in the reflected wavelengths of the FBGs are observed. Starting at $20 \mathrm{~K}$, temperature measurement with an accuracy of $\pm 1 \mathrm{~K}$ should be possible. Additional investigations will reveal whether the temperature of liquid hydrogen (boiling point of $20.3 \mathrm{~K}$ ) will be able to be monitored. Multipoint sensors based on FBGs are clearly an option for applications in the field of liquid nitrogen and liquefied natural gas, which require temperatures of $77 \mathrm{~K}$ and $111 \mathrm{~K}$ respectively, to be measured.

At ITEP, four glass fibers with different types of FBGs were inserted in bores drilled in a copper block. Several ceramic cryogenic temperature sensors (TVOs) were used as reference. The spectra of the gratings and the calculated Bragg wavelengths were evaluated using a Siemens SITRANS TO500. Although, a vacuum is typically created inside the cryostat, in this case, the pressure inside the chamber was kept at atmospheric pressure to avoid changes of the reflected wavelength due to a low pressure exerted on the fiber. The chamber itself was cooled down to $8 \mathrm{~K}$ within less than an hour by placing small portions of liquid helium inside it. Using a heat exchanger and a combination of liquid helium cooling and liquid nitrogen cooling, the gratings were warmed up to room temperature over a period of several days. The cryostat, the automated cooling system and the interrogator are shown in Fig. 2.

The sensitivity of FBGs regarding temperature changes was investigated in the temperature range from $8 \mathrm{~K}$ up to $250 \mathrm{~K}$ at ITEP - and from $-40^{\circ} \mathrm{C}$ up to $650^{\circ} \mathrm{C}$ in our laboratory. The change in wavelength at room temperature is approximately $11 \mathrm{pm} / \mathrm{K}$ rising to about $14 \mathrm{pm} / \mathrm{K}$ at $600{ }^{\circ} \mathrm{C}$. The transfer function for low temperatures (Fig. 3) reveals a sensitivity of 
approximately $0 \mathrm{pm} / \mathrm{K}$ in the temperature range between $8 \mathrm{~K}$ and $15 \mathrm{~K}$. Starting at $20 \mathrm{~K}$, the sensitivity seems to be high enough to allow temperatures to be monitored.

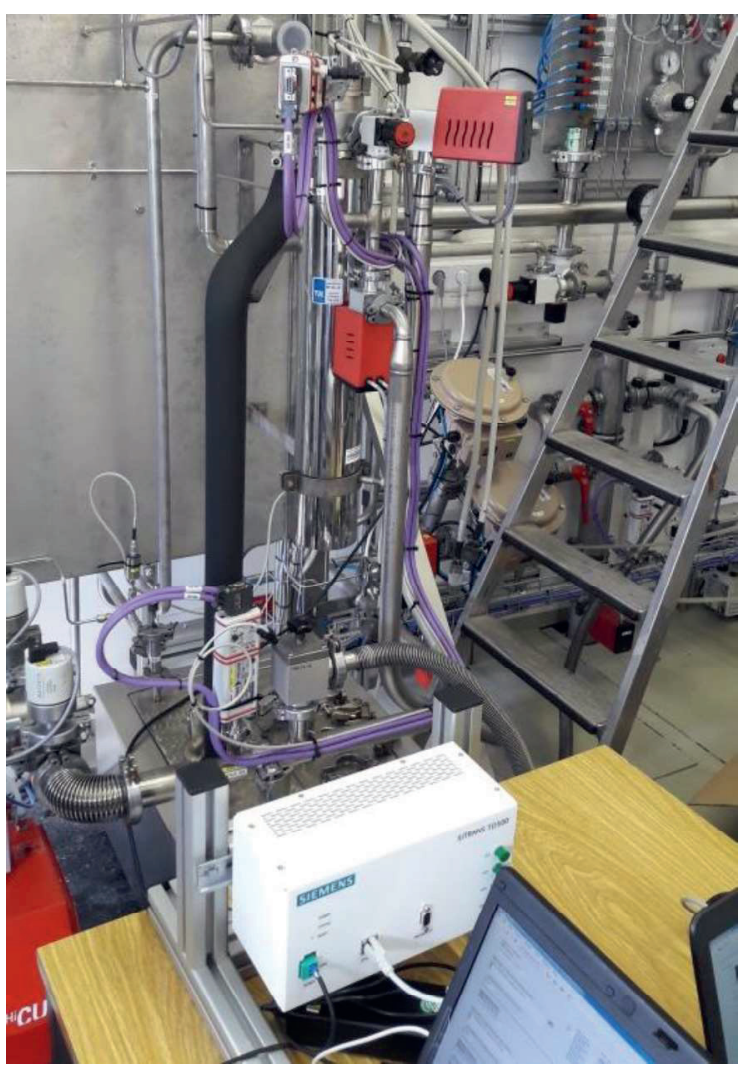

Fig. 2. Low temperature measurements with FBGs with a cryostat at ITEP, KIT-Nord.

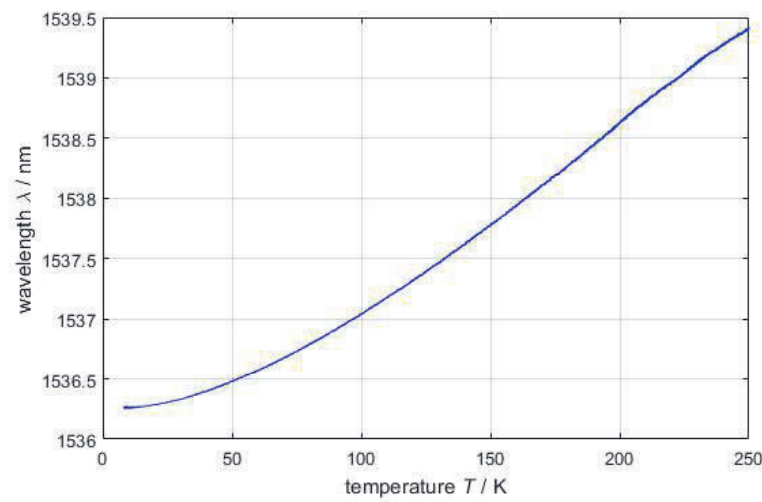

Fig. 3. Transfer function of a fiber Bragg grating. The graph shows the Bragg wavelength of the FBG for a temperature range from $8 \mathrm{~K}$ to $250 \mathrm{~K}$ for one of the specimens.

\section{Temperature Monitoring}

Several industrial applications, e.g. grain mills, require that temperatures up to $200{ }^{\circ} \mathrm{C}$ are monitored. For these types of applications, the accuracy requirements are much lower than for temperature measurements used in process control systems, e.g. for chemical applications. Draw tower gratings with a special coating allow temperatures to be monitored at multiple points and - due to the flexibility of the fiber and lengths of up to hundreds of meters - they enjoy a high degree of versatility (Fig. 1 (right)). As discussed in the previous paragraph, multipoint temperature sensors based on FBGs are also an excellent choice for monitoring the temperature of liquid nitrogen, LNG and possibly even liquid hydrogen.

\section{Temperature Measurement for Process Control}

Temperature measurement and process control by - at least partly - controlling the temperature play an important role in a large variety of applications in the oil\&gas, chemical, pharmaceutical and food\&beverage sectors. New developments in chemical plant designs have led to the introduction of tube reactors. These reactors are used by chemical design engineers in small scale production setups with a height of less than a meter in laboratory environments up to heights of more than $20 \mathrm{~m}$ in production plants. Laboratory and pilot tests have proven excellent long-term stability for temperatures up to $450{ }^{\circ} \mathrm{C}$, temperature calibration in the factory without any need for recalibration or adjustment at the customers production site for type I gratings in photosensitive fibers and for type II gratings (see Fig. 4 for an example of long-term stability). The glass fibers are housed in flexible stainless steel capillaries (316L) to withstand harsh environments. As a result of a thin plug at the top of the lance, the lance may stay inside the vessel or inside a protective conduit during inspection and maintenance. The interrogator features a depolarizer to compensate the polarization dependency of the reflected wavelength of gratings written with femtosecond lasers.

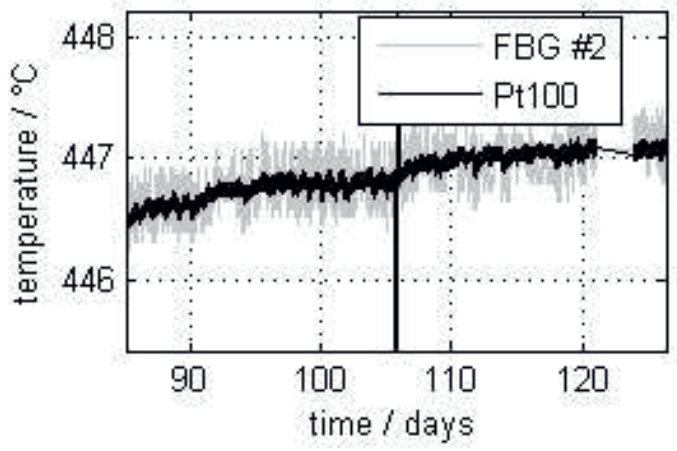

Fig. 4. Long-term stability of a type II grating at a temperature of $450^{\circ} \mathrm{C}$. Temperature calibration is performed using a slow temperature ramp from $50{ }^{\circ} \mathrm{C}$ up to $450{ }^{\circ} \mathrm{C}$ and vice versa (Fig. 7). The hysteresis is within the relative measurement uncertainty of $\pm 0.3 \mathrm{~K}$ (mainly due to the effects of the depolarizer). The averaged offset after 125 days at $450{ }^{\circ} \mathrm{C}$ and at $50{ }^{\circ} \mathrm{C}$ is less than $0.2 \mathrm{~K}$. 


\section{Advances with Femtosecond Gratings}

Previously, a university would be able to write a single grating per day. However, in recent years, with the advances made in femtosecondlaser-written gratings, fully automated production lines are now capable of producing several gratings per minute. This has driven the production cost down to an attractive level for temperature measurement applications. Tests in our laboratories have shown that femtosecond gratings can withstand temperatures up to $1050{ }^{\circ} \mathrm{C} / 1100{ }^{\circ} \mathrm{C}$ in standard telecommunication glass fibers such as Corning ${ }^{\circledR}$ SMF-28 ${ }^{\circledR}$. Draw tower gratings and phase mask type I gratings disappear at temperatures of about $300^{\circ} \mathrm{C}$ and $650^{\circ} \mathrm{C}$, respectively. Excellent long-term stability for temperatures up to $450{ }^{\circ} \mathrm{C}$ can be achieved (see Fig. 4).

Measurements in environments up to $800{ }^{\circ} \mathrm{C}$ seem to be feasible. Up until now, the long-term stability at such extremely high temperatures is not sufficient for accurate temperature measurements. Nevertheless, for special applications such as chemical design engineering, if the temperature distribution is of interest, multi-point temperature measurements with FGBs allow chemical reactions to be investigated resulting in important insights.

Recently, FBGs that are not measurably influenced by changes of the polarization have been able to be produced. These advances will lead to stable, high-temperature multipoint measurement systems with reduced noise without the necessity of integrating a depolarizer into the interrogator.

\section{Annealing and Long-term Stability}

Due to the amorphous structure of glass and the structural change of the fiber due to writing the gratings, it can be expected that FBGs will undergo a long-term relaxation process, which may cause changes in the reflected wavelength and thus lead to deviations (drift) in temperature measurement.

In order to prevent drift at the customer's site, annealing the glass fiber is a mandatory production step for high temperature measurements.

A wide combination of type I and type II gratings, different glass fibers (including Corning ${ }^{\circledR}$ SMF- $28^{\circledR}$ ) and annealing recipes have been tested for long-term stability. Short and long pre-treatment (ranging from several minutes to several hours) at high temperatures (up to $1100{ }^{\circ} \mathrm{C}$ ) and different cooling strategies have been applied [3, 4]. The long-term stability at $450{ }^{\circ} \mathrm{C}$ has been thoroughly investigated over several months. Depending on the combination of the type of the grating, the glass fiber and the annealing recipe, excellent longterm stability (see Fig. 4 and Fig. 5), but also drifts of several Kelvin/month have been observed.

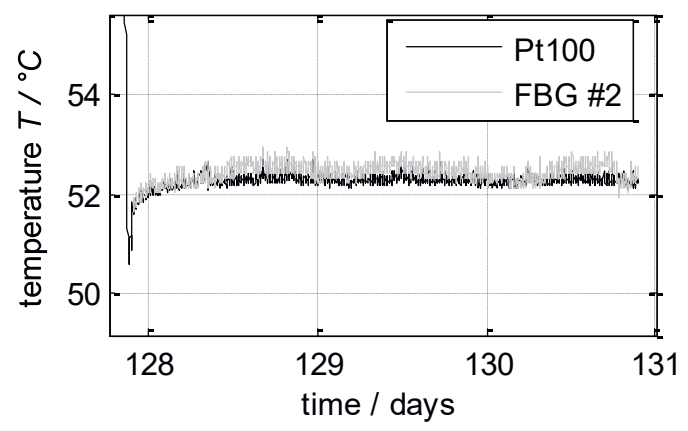

Fig. 5. Long-term stability of a type II fiber Bragg grating. The multi-point temperature measurement lance had been tested at a temperature of $450{ }^{\circ} \mathrm{C}$ for more than $1500 \mathrm{~h}$. The offset at $50^{\circ} \mathrm{C}$ is less than $0.2 \mathrm{~K}$.

Annealing of long lances with lengths of up to $40 \mathrm{~m}$ requires a huge furnace. Lances with a length of $12 \mathrm{~m}$ have been annealed in a furnace at Majolika Karlsruhe, Germany, a ceramic manufacturer (see Fig. 6). The lances were put into the furnace in a ring. The temperature was increased by $1 \mathrm{~K} / \mathrm{min}$ until the final annealing temperature had been reached.

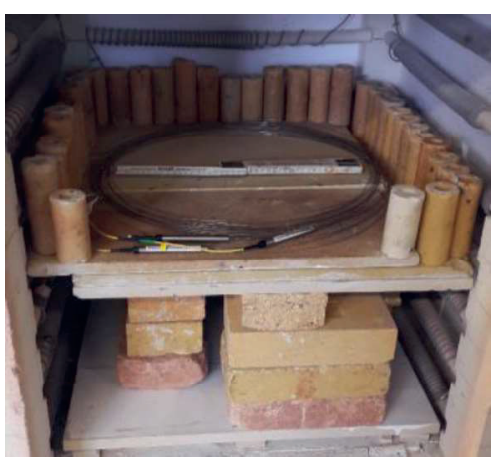

Fig. 6. Annealing type II gratings in a furnace at Majolika Karlsruhe, Germany.

\section{Transfer Function}

In order to fulfill the requirements in process industries, multipoint temperature measurement lances have to be calibrated based on normalized standards as they are provided, e.g. by the Physikalisch-Technische Bundesanstalt. Evaluating the repeatability and relative accuracy compared to calibrated platinum resistance thermometers is a first important step towards certified calibration.

Measuring the transfer function for each grating separately is always a potential measure to calibrate a grating. However, this process is very elaborate and cost intensive. Thus, it is an 
important objective to derive calibration transfer functions that are valid for at least a complete production batch. For type I gratings, it is known that the transfer function is valid for a whole batch for all wavelengths. For type II gratings, laboratory measurements are an on-going process. To measure the transfer functions, temperatures have been changed very slowly (see Fig. 7). The transfer function derived for one of the gratings is then compared with gratings with different center wavelengths and gratings produced in glass fibers from other batches.

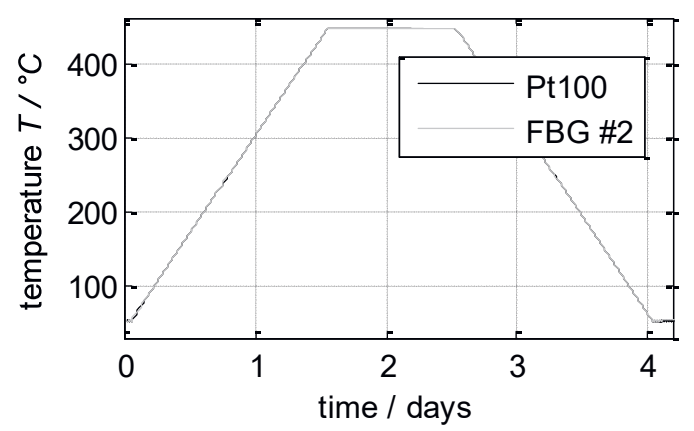

Fig. 7. Slow temperature ramp to evaluate the transfer function. The RTD (reference thermometer) and the specimen under test are both linked to a copper bar, which has notches for the RTD and the multipoint temperature measurement lance.

\section{Summary}

In recent years, important advances have been made regarding spatially distributed temperature measurements based on fiber optical sensors for all kinds of applications in the process industry. These advances lead to factory calibrated plug \& play systems, which replace conventional multipoint temperature measurements for temperature monitoring and process control. The measurement system is attractive for all kinds of applications, involving very low up to high temperatures $(30 \mathrm{~K}$ to possibly $800^{\circ} \mathrm{C}$ ), hazardous environments and systems with strong electromagnetic waves. This approach is also attractive as a result of the highly flexible configurations due to the bendable glass fiber and steel capillary, low cabling costs over distances of several hundred meters via a single standard telecommunication glass fiber. Further, the measurement system is rugged with a low profile plug mounted at the top of the lance affording an IP68 degree of protection, which allows the system to be seamlessly integrated into the customer's distributed control system via PROFIBUS DP.

\section{References}

[1] von Dosky, Stefan, et.al., Proceedings AMA Conferences 2013, 578-582 (2013); doi: 10.5162/sensor2013/D6.3

[2] R. Kashyap, Fiber Bragg Gratings, 2nd Edition, Academic Press, 2009; ISBN 9780123725790

[3] F. K. Coradin et.al., Long-term stability decay of standard and regenerated Bragg gratings tailored for high temperature operation, Journal of Microwaves, Optoelectronics and Electromagnetic Applications, Vol. 12, No. 2, 719729 (2013)

[4] Yuhua Li et.al., Fiber Bragg Grating for High Temperature Applications, 15th OptoElectronics and Communications Conference (OECC2010) Technical Digest, July 2010, Sapporo Convention Center, Japan 